\title{
CHARACTERISTICS AND RISK FACTORS OF CHRONIC POST-TRAUMATIC HEADACHE AFTER MILD HEAD INJURY- A STUDY IN A TERTIARY CARE CENTRE IN EASTERN INDIA
}

\author{
Ramesh Bhattacharyya ${ }^{1}$, Shantanu Ghosh², Kartick Chandra Ghosh ${ }^{3}$, Sarbajit Ghosh ${ }^{4}$, Suman Das ${ }^{5}$, Hema Krishna Pattem6, \\ Gouranga Prasad Mondal ${ }^{7}$
}

\author{
${ }^{1}$ Associate Professor, Department of Neurology, CNMC, Kolkata, West Bengal, India. \\ ${ }^{2}$ Assistant Professor, Department of Neurosurgery, CNMC, Kolkata, West Bengal, India. \\ ${ }^{3}$ Associate Professor, Department of Neurology, CNMC, Kolkata, West Bengal, India. \\ ${ }^{4}$ Post Doctoral Resident, Department of Neurology, CNMC, Kolkata, West Bengal, India. \\ 5 Post Doctoral Resident, Department of Neurology, CNMC, Kolkata, West Bengal, India. \\ ${ }^{6}$ Post Doctoral Resident, Department of Neurology, CNMC, Kolkata, West Bengal, India. \\ ${ }^{7}$ Professor, Department of Neurology, CNMC, Kolkata, West Bengal, India.
}

\section{BACKGROUND}

ABSTRACT

Traumatic head injury is one of the most important public health problems. $75 \%$ of all head injuries are mild. Leading causes of head injuries are road traffic accident, falls, assaults, colliding with moving or stationary objects. Headache is one of the most common symptoms after mild head injuries (MHI). When headache starts within 7 days of head injury, it is called as post traumatic headache (PTH). PTH occurs in 30-90\% of patients with MHI, majority of which resolves within a few months, but when it persists beyond 3 months- it is termed as chronic PTH (cPTH). Trauma to the extracranial and intracranial structures produces neurogenic inflammation-leading to both peripheral and central sensitization. They not only produce headache but also help in the perpetuation of pain. Majority of CPTH are tension type headache (TTH) and migraine. Others like medication overuse headache $(\mathrm{MOH})$, coexisting migraine and TTH, cluster headache, occipital neuralgia, hemicrania continua, supraorbital neuralgia are rare. Diagnosis of cPTH is mainly clinical. Investigations are helpful to exclude other secondary causes. Some risk factors like female gender, poor socioeconomic status, past history and family history of headache are associated with cPTH. Aim of the study is to identify the characteristics and risk factors for CPTH in our population.

\section{METHODS}

This is a cross sectional observational study that included patients attending outdoor or admitted in the indoor of Neurology and Neurosurgery departments of $\mathrm{CNMCH}$ with headache that persisted more than 3 months of MHI. The study period was from 1.8.2016 to 1.7.2018. MHI were diagnosed by emergency and neurosurgery department of our hospitals as those patients who presented within 24 hours with any of the criteria laid down by ICHD3 for MHI. Exclusion criteria were those below 15 years and above 65 years of age, moderate or severe head injury, whiplash injury, MHI with subsequent deterioration, clinical features and imaging suggestive of any other secondary causes of headache. Detailed history was taken, and neurological examination was done to detect the type of headache disorder and exclude other secondary headache disorders. Routine blood tests and CT scan of brain followed by MRI brain were done for every patient while LP and EEG were done in selected cases. In some cases, indomethacin response (Hemicrania continua), nerve block (Occipital, supra orbital neuralgia) was done for confirmation of diagnosis. All data were noted and analysed. This was a cross sectional-observational study and sample size was 91 .

\section{RESULTS}

Most common types of cPTH were TTH and migraine. Other rare types were occipital neuralgia and supraorbital neuralgia. Chronic TTH is more common than episodic TTH in this sample. Chronic migraine seems to be higher in this patient group compared with non-traumatic migraine. Road traffic accident is still the major cause of head injury. Risk factors for chronic post traumatic headache are female gender, poor literacy rate, higher unemployment rate, poor socioeconomic status and family history of headache.

\section{CONCLUSIONS}

Mild head injury may cause a lot of morbidity including various types of headache disorders. Identification of headache disorder is important as the patient can be educated about their illness and proper treatment can be administered, to alleviate their symptoms which can be disabling.

HOW TO CITE THIS ARTICLE: Bhattacharyya R, Ghosh S, Ghosh KC, et al. Characteristics and risk factors of chronic post-traumatic headache after mild head injury- a study in a tertiary care centre in Eastern India. J. Evolution Med. Dent. Sci. 2019;8(17):13761380, DOI: $10.14260 /$ jemds/2019/306

'Financial or Other Competing Interest': None.

Submission 13-03-2019, Peer Review 17-04-2019,

Acceptance 23-04-2019, Published 29-04-2019.

Corresponding Author:

Dr. Shantanu Ghosh,

C/4/49, Kendriya Vihar,

Kolkata-700052, West Bengal, India.

E-mail: shashwata_shantanu@yahoo.co.in

DOI: $10.14260 /$ jemds $/ 2019 / 306$

(c) $(1)$

\section{BACKGROUND}

Head injury is any sort of injury to the brain, skull or scalp. The number of new cases in India is gradually increasing. The incidence rate is about $150 / 1,00,000.175 \%$ of all head injuries are mild. ${ }^{2}$ Headache is pain in any region of the head. To call it as post traumatic headache (PTH), headache should start within 7 days after the injury. ${ }^{3}$ Headache after injury may be a new primary headache or exacerbation of preexisting primary headache. PTH is estimated to affect $30-90 \%$ of patients with mild head injury (MHI). ${ }^{4}$ So it is an important 
public health problem in India. Most PTH resolves within 6 months, but $25 \%$ persists. ${ }^{5}$ PTH is most prevalent and lasts longer after MHI. PTH is regarded as chronic (cPTH), when it continues for more than 3 months after incurrence of injury. ${ }^{6}$ Prevalence of cPTH is $47-95 \%$ in MHI, compared to $20-30 \%$ in moderate to severe head injury.7 Pathogenesis of PTH is not clearly known. Damage to pain sensitive extracranial (Muscles) or intracranial (Dural vessels) structures leads to neurogenic inflammation by cytokines, chemokines and sensitization of pain transmission. Damage to spinothalamicthalamocortical pathways results in alteration of central modulatory control over nociceptive inputs. ${ }^{8}$ Damage to inhibitory control of pain increases pain transmission. Additional factors are active like post-traumatic stress disorder (Anxiety, depression) that can modulate pain pathways. Peripheral and central sensitization may contribute to perpetuation of pain in chronic post traumatic headache. ${ }^{9}$ Patient expectation of post traumatic pain and underlying genetic predisposition can also influence headache. ${ }^{10}$ Majority of cPTH are of 2 types- tension type (TTH) or migraine. Migraine with aura (MA) is less common than migraine without aura (MO). Both TTH and MO can be divided again to episodic and chronic variety. They can be called as chronic when headache is on for 15 days or more per month, for more than 3 months in the absence of analgesic medication overuse. ${ }^{3}$

Diagnosis of cPTH is mainly clinical by history and examination. Investigations are for excluding other secondary causes. Routine investigations including CT scan of brain and MRI are normal, but advance neuroimaging technique such as fast sequence MRI, diffuse tensor imaging show structural changes in regions including frontal and temporal lobes, thalamus and corpus callosum. ${ }^{11}$

Some risk factors like female gender, poor socioeconomic status, past history and family history of headache are associated with cPTH. Aim of the study is to identify the characteristics and risk factors for $\mathrm{CPTH}$ after MHI.

\section{METHODS}

This cross-sectional observation study includes patients attending the outdoor and admitted in indoor of Neurology and Neurosurgery Departments of Calcutta National Medical College and Hospital with headache that persisted more than 3 months after MHI. The study period was from 01-08-2016 to $01-07-18$. The total number of patients who completed this study was 91. MHI were diagnosed by Emergency \& Neurosurgery Department of our Hospital, who presented to the ED within 24 hours post injury with any of the following features -loss of consciousness less than $30 \mathrm{~min}$., Glasgow Coma Scale (GCS) 13-15 and symptoms and signs of concussion. Inclusion criteria for this study were headaches which started within 7 days after MHI and that persisted more than 3 months. Exclusion criteria were patients below 15 years of age and above 65 years of age, moderate/severe injury, whiplash injury, MHI with subsequent deterioration, ongoing symptoms after mild head injury suggestive of secondary cause like low CSF pressure headache, scalp laceration, local trauma, subdural hematomas, extradural hematomas, carotid or vertebral artery dissection or cerebral vein thrombosis.

\begin{tabular}{|c|c|c|}
\hline Type of Head Ache & $\begin{array}{c}\text { Percentage } \\
(\%)\end{array}$ & $\begin{array}{l}\text { Total Number } \\
\text { and (Gender) }\end{array}$ \\
\hline Tension type headache (TTH) & $69.2 \%$ & $63(\mathrm{~F}-43)$ \\
\hline Migraine without aura (MO) & $17.5 \%$ & $16(\mathrm{~F}-11)$ \\
\hline $\begin{array}{c}\text { Medication over use headache } \\
\text { (MOH) }\end{array}$ & $3.2 \%$ & $3(\mathrm{~F}-1)$ \\
\hline $\begin{array}{l}\text { Migraine and TTH } \\
\text { (Coexisting) }\end{array}$ & $3.2 \%$ & $3(\mathrm{~F}-1)$ \\
\hline Cluster headache & $2.1 \%$ & $2(\mathrm{~F}-0)$ \\
\hline Occipital neuralgia & $2.1 \%$ & $2(\mathrm{~F}-0$ \\
\hline Hemicrania continua & $1.09 \%$ & $1(\mathrm{~F}-0)$ \\
\hline Supra orbital neuralgia & $1.09 \%$ & $1(\mathrm{~F}-0)$ \\
\hline \multicolumn{3}{|c|}{ Table 1. Classification of Headache } \\
\hline
\end{tabular}

Cases where history and examination fit with ICHD-3 criteria of $\mathrm{CPTH}$ attributed to $\mathrm{MHI}^{3}$ were included.

A. Headache with typical characteristics known fulfilling $\mathrm{C}$ and D

B. Head trauma with all the following.

1. Either no loss of consciousness or loss of consciousness $<30 \mathrm{~min}$. duration.

2. Glasgow coma scale $(\mathrm{GCS})>13$.

3. Symptoms and / or signs diagnostic of concussion.

C. Headache develops within 7 days after head trauma.

D. Headache persists more than 3 months after head trauma.

Symptoms and signs diagnostic of concussion includes headache, confusion, lack of coordination, memory loss, nausea, vomiting, dizziness, ringing of ears, sleepiness and excessive fatigue and it usually recovers within 24 hours.

Full neurological examination was undertaken for all cases of post-traumatic headache including blood pressure management and fundoscopy to exclude secondary cause of headache.

Investigation was done to exclude secondary causes. Blood tests including full blood count, ESR, CRP, creatinine, Calcium, thyroid function test LFT and in some cases VDRL, HIV, Lyme antibodies, ANA Profile were done. Neuro imaging including plain x-ray, CT of head, MRI Brain in all cases and Lumbar puncture, Electroencephalography in some cases were done.

All the data are grouped and analysed accordingly.

\section{RESULTS}

Total number of patients following inclusion and exclusion criteria on and from 01.08.16 to 01.07.18 were 91, of which females were $61.5 \%(n=56)$. Types of headache noted were Tension Type headache 69.2\% ( $\mathrm{n}=63)$, Migraine 17.5( $\mathrm{n}=16)$, cluster headache 2.1( $\mathrm{n}=2)$, hemicrania continua $1.09 \%(\mathrm{n}=1)$, medication over use headache $(\mathrm{MOH}) 3.2 \%(\mathrm{n}=3)$, occipital Neuralgia 2.1\% $(n=2)$, supra orbital neuralgia 1.09\% $(n=1)$, coexisting migraine and TTH $3.2 \%(n=3)$.

Distribution of headache in TTH were generalized in $50.7 \%(n=32)$, occipital in $34.9 \%(n=22)$ and frontal in $14.2 \%$ $(\mathrm{n}=9)$. Distribution of headache in migraine were hemicranial and side locked to the side of trauma in $50 \%(n=8)$, changing side $18.7 \%(n=3)$ and generalized $31.2 \%(n=5)$. 


\begin{tabular}{|c|c|c|}
\hline Area & Percentage & No. \\
\hline Generalised & $50.7 \%$ & 32 \\
\hline Occipital & $34.9 \%$ & 22 \\
\hline Frontal & $14.2 \%$ & 9 \\
\hline \multicolumn{2}{|c|}{ Table 2. Distribution of Tension Type Headache } \\
\hline
\end{tabular}

Recurrence pattern of headache among TTH patients $(n=63)$ observed were infrequent TTH 39.6\% ( $n=25)$ Chronic TTH $(n=21) 33.3 \%$ and frequent TTH $26.9 \%(n=17)$.

Recurrence pattern of headache among MO patients ( $n=16)$ observed were episodic MO 68.7 (n-11) chronic MO $31.2 \%(n=5)$.

\begin{tabular}{|c|c|c|}
\hline TTH $(n=63)$ & Percentage \% & Number \\
\hline $\begin{array}{c}-\quad \text { Infreque } \\
\text { nt }\end{array}$ & $39.6 \%$ & 25 \\
\hline - Chronic & $33.3 \%$ & 21 \\
\hline $\begin{array}{ll}- & \text { Frequen } \\
\mathrm{t} & \end{array}$ & $26.9 \%$ & 17 \\
\hline Migraine ( $n=16)$ & Percentage \% & Number \\
\hline - Episodic & $68.7 \%$ & 11 \\
\hline - Chronic & $31.2 \%$ & 5 \\
\hline \multicolumn{3}{|c|}{ Table 3. Recurrence Pattern of TTH \& MO } \\
\hline
\end{tabular}

Various causes of head injury were Road traffic accident (RTA) $51.6 \%(n=47)$ fall $19.7 \%(n=16)$ assault $17.5 \%(n=16)$, self-injury $3.2 \%(n=3)$, colliding with moving or stationary objects $7.6 \%(n=7)$.

\begin{tabular}{|c|c|c|c|}
\hline Cause & Percentage & Number & (M / F) \\
\hline Road Traffic Accident & $51.6 \%$ & 47 & $(27 / 20)$ \\
\hline Fall & $19.7 \%$ & 18 & $(5 / 13)$ \\
\hline Assault & $17.5 \%$ & 16 & $(3 / 13)$ \\
\hline Self-injury & $3.2 \%$ & 3 & $(0 / 03)$ \\
\hline $\begin{array}{c}\text { Colliding with moving } \\
\text { on stationary objects }\end{array}$ & $7.6 \%$ & 7 & $(2 / 5)$ \\
\hline
\end{tabular}

Table 4. Causes of Head Injury

Factors that have significant impact on chronic post traumatic headache were Female gender 61.5\% ( $n=56)$, low literacy rate $58.2 \%(\mathrm{n}=53)$, unemployment rate $10.9 \%$ $(\mathrm{n}=10)$ socio-economic status people holding card of below poverty line BPL $51.6 \%(n=47)$. Past history of episodic TTH $60.3 \%(n=38)$ in chronic post traumatic TTH group and past history of episodic migraine were seen in $18.7 \%(n=3)$ of chronic post traumatic migraine group. Family history of TTH in post-traumatic TTH group were $47.6 \%(n=30)$ and family history of migraine in chronic post traumatic migraine group $75 \%(n=12)$.

\section{DISCUSSION}

The International Headache Society IHS criteria $^{3}$ require headache onset within 1 week of head injury or of regaining consciousness. However, in clinical practice it is often difficult to determine when the headache actually started since headache may be mild and other pains like neck pain is more prominent. In the general population TTH is the most frequent headache usually bilateral pressing of mild or moderate intensity and not associated with nausea or vomiting, no more than one of photophobia or phonophobia. After mild head injury most frequently $85 \%$ seen pattern that resembles TTH.(12) In one study headaches were mild in 30\%, moderate in $52 \%$, severe in $18 \%$ and the pain was occipital in
$51 \%$ of patients, frontal in $44 \%$ and generalized in $11 \%$ of patients.(13) In this study occurrence of chronic post traumatic TTH was $69.2 \%(n=63)$ in females. Distribution of pain was generalized in $50.7 \%(n=32)$, occipital $34.9 \%$ $(n=22)$ and frontal $14.2 \%(n=9)$. TTH are featureless so patients are often reluctant to approach health care professional. TTH are generalized but area of trauma over the skull can dictate distribution of pain.

IHS(3) classification of TTH according to recurrence are infrequent episodic TTH if at least 10 episodes occurring <one per month on average (12 days / year). Frequent Episodic TTH at least 10 episodes occurring on $>1$ but $<15$ days per month for at least 3 months ( $>12$ days and $<180$ per year) and chronic TTH if headache occurring on $>15$ days / month on average for $>3$ months ( $>180$ days / year). In a population based study overall prevalence of episodic TTH was $38.3 \%$ and Chronic TTH was $2.2 \%{ }^{14}$ In this study among all the patients who suffered from Chronic post traumatic TTH, 39.6\% (n=25) had infrequent episodic TTH, 33.3\% $(n=21)$ had frequent episodic TTH and 26.9\% (n=17) had Chronic TTH. In one study chronic TTH after mild head injury was $40 \% .{ }^{15}$ As it was a special group of patients who suffered from mild head injury it was bound to be higher.

Migraine is characterized by recurrent, hemicranial pulsating, moderate to severe headaches lasting for 2-72 hours associated with nausea, vomiting, photophobia, phonophobia, osmophobia and disability. Chronic migraine is defined as migrainous headache for 15 days or more per month for more than 3 months in the absence of analgesic medication overuse. In a study, incidence of post traumatic migraine was of $28.6 \% .^{16}$ In a population-based study prevalence of chronic migraine was in the range of 1.4$2.2 \% 17$. In one study, migraine after mild head injury was $26.6 \% 15$. In this study $17.5 \%(n=16)$ suffered from posttraumatic migraine. Distribution of headache is hemicranial and side locked to the side of trauma in $50 \%(n=8)$, changing sides in $18 \%(n=3)$ and generalized in $31.2 \%(n=5)$. Very few studies have assessed the side locked character in migraineurs. Sjaastadet $\mathrm{al}^{18}$ observed strictly unilateral headache in $42 \%$ of non-traumatic migraineurs. In this study among all those with chronic post traumatic migraine, $68.7 \%$ $(n=11)$ suffered from episodic migraine and the rest $31.2 \%$ $(n=5)$ suffered from chronic migraine. In episodic migraine headache attacks were 2-3 attacks per month.

Medication over use headache is headache that occurs on $>15$ days/month associated with frequent use of simple analgesics $>15$ days / month or triptans> 10 days or ergotamine $>10$ days/month for at least 3 months. It may be chronic TTH or chronic migraine depending upon the initial headache type. One study showed analgesic abuse headache to be of $6.6 \% 15$. In this study, it was $3.2 \%(n=3)$. None of these patients were found to be overusing ergotamine or triptans.

Patients who suffered from both chronic TTH and migraine previously called as mixed headache are now identified as co-existing migraine and TTH. In this study coexisting migraine and TTH were seen in $3.2 \%(n=3)$ of these cases.

Cluster headache is an excruciating painful condition strictly unilateral having cranial autonomic symptoms. It occurs with circannual and circadian periodicity. The male: female ratio is 2.5-7.2:1. Cluster headache can occur after mild head injury. One study showed cluster headache to be of 
$10 \%{ }^{19}$. Some of these headaches may not undergo remission. In this study it was $2.1 \%(\mathrm{n}=2)$ and all were male.

Occipital neuralgia is characterized by pain usually deep and aching in the distribution of second cervical dorsal root that can be relieved by nerve block ${ }^{20}$. Characteristic pain may be paroxysmal and jabbing as stated by HIS. Greater, lesser or third occipital nerve may be involved. They can occur after mild head injury. The aching, pressure, stabbing, paroxysmal or jabbing pain can occur in nuchal-occipital distribution. In this study occipital neuralgia occurred in $2.1 \%(n=2)$. All were male and all are in the distribution of greater occipital nerve.

Hemicrania continua is a syndrome of continual pain on one side of the head, associated with ipsilateral cranial autonomic symptoms and migrainous features like photophobia or phonophobia. It is highly responsive to indomethacin. In this study only one case (1.09\%) was diagnosed as hemicrania continua.

Supra orbital neuralgia is characterized by forehead pain in the territory supplied by supra orbital nerve, tenderness over supra orbital notch or over the nerve route and absolute relief of symptoms by supra orbital nerve block. In this study only one male patient had supraorbital neuralgia.

Head injury is any trauma to the scalp, skull or brain. Traumatic brain injuries are one of the important public health problems in the world. According to centre for disease control and prevention in 2003 falls (32\%) are the most frequent cause of traumatic brain injury with motor vehicle accident being second(19\%)followed by struck by/against events(18\%),assault (10\%)and others $11 \%$.

One study in India showed road traffic accident in 60\%, fall in $25 \%$ and assault in $10 \%{ }^{1}$. Traumatic brain injury from falls are the most common cause in the very young and elderly. TBI from motor vehicle accident is most common in the 15-19 years of age. Males are about 1.5 times more likely than females to sustain a traumatic brain injury although females most commonly suffer from PTH

Road Traffic accidents occurs when a vehicle that is moving along a roadway collides with another vehicle or object. In this study among all the patients having mild head injury RTA was 51.6\% (M-27/ F-20). Moving from a higher to lower level, typically rapidly and without control fall occurred in $19.7 \%$ (M-5/ F-13). Assault is physical attack by someone causing bodily harm. Number of assault cases were $17.5 \%$ (M-3/ F-13). Deliberately hurting own self is called self-injury. Self-injury was found to be $3.2 \%$ (F-3). Colliding with moving or stationary objects was found to be $7.6 \%$ (Male-2/ Female-5).

There are some injury related phenomenon that can predict chronicity of headache. ${ }^{21}$ They are presence of headache dizziness or nausea in emergency room directly following mild head injury, duration of post traumatic amnesia, dizziness or fatigue or poor vision at 2 weeks post injury. There are some risk factors for chronic post traumatic headache. Female gender increases the chances of Chronic post traumatic headache either TTH or migraine. ${ }^{22}$ In this study females were $61.5 \%(n=56)$, among which TTH $47.2 \%$ $(n=43)$ and migraine $12 \%(n=11)$. The ability to read and write is called literacy. Total number of literate persons in a given age group expressed as a percentage of the total population in that age. The adult literacy rate measures literacy among persons aged 15 years and above. Lower literacy rate increases chance of cPTH. Literacy rate of the general population of West Bengal in 2011 is $77.08 \% .^{23}$ In this study literacy rate was $58.2 \% \quad(n=53)$. Majority of patients were coming from urban slums and nearby districts. Unemployment or joblessness is the situation of actively looking for employment but not currently employed. Unemployment rate in West Bengal is $4.9 \% .^{24}$ In this study unemployed persons are 10.9\% (n-10, M-9/ F-1). Socioeconomic status is economic and social position of an individual or family in relation to others based on income, education and occupation. Those whose annual income is below Rupees 27,000 per annum are considered below poverty line and hold BPL card. $22 \%$ of Indian people are below the poverty line. ${ }^{25}$ In this study people of BPL were $51.6 \%(n=47)$ which is higher than the general population. It confirms that lower socioeconomic status increases chance of Chronic Post Traumatic Headache.

Prevalence of Migraine is 7.7\% and TTH 52\% in general population. Presence of pre injury headache is a risk factor of

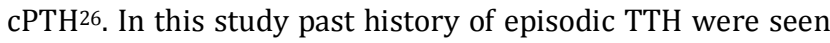
in $60.3 \%(n=38)$ of $\mathrm{CPTH}$ of TTH group and past history of episodic migraine were seen $18.7 \%(n=17) \mathrm{cPTH}$ of migraine group.

Family history TTH in non-traumatic TTH is found in $40 \%$ and first degree relative had a 2.1 to 3.9-fold increased risk of TTH compared to general population 27 . In our study family history TTH in cPTH of TTH group were $47.6 \%(n=30)$. Family history of migraine in non-traumatic migraine is found to be $70 \%$ and first degree relative had a 14 -fold increase risk of migraine compared to general population ${ }^{28}$. In our study family history of migraine in CPTH of migraine group were $75 \%(n=12)$. So, family history of TTH and migraine are risk factors for developing $\mathrm{CPTH}$.

\section{CONCLUSIONS}

Headache can persist for more than 3 months after mild head injury. This headache is called as cPTH. Types of cPTH are many. They can mimic primary headache disorders. Common varieties are TTH and migraine, while others including $\mathrm{MOH}$, coexisting migraine and TTH, cluster headache, occipital neuralgia, hemicrania continua, and supra orbital neuralgia are rare. cTTH and migraine are higher in female. TTH are mostly generalized.

\section{REFERENCES}

[1] Gururaj G. Epidemiology of traumatic brain injuries: Indian scenario. Neurol Res 2002;24(1):24-8.

[2] Lee LS, Shih YH, Chiu WT, et al. Epidemiologic study of head injuries in Taipei city, Taiwan. Zhonghua Yi Yue Za Zhi (Taipei) 1992;50(3):219-25.

[3] Headache classification committee of the International Headache Society (IHS). The international classification of headache disorders $3 \mathrm{rd}$ edition. Cephalgia 2018;38 (1):1-211.

[4] Thornhills S, Teasdale GM, Murray GD, et al. Disability in young people and adults one year after head injury: prospective cohort study. BMJ 2000;320(7250):16315.

[5] Yang CC, Hua MS, Tu YK, et al. Early clinical characteristics of patient with persistent postconcussion symptoms: a prospective study. Brain Inj 2009;23(4):299-306. 
[6] Baandrup L, Jensen R. Chronic post traumatic headache--a clinical analysis in relation to international classification 2nd edition. Cephalalgia 2005;25(2):132-8.

[7] Hoffman JM, Lucas S, Dikmen S, et al. Natural history of headache after traumatic brain injury. J Neurotrauma 2011;28(9):1719-25.

[8] Defrin R, Gruener H, Schreiber S, et al. Quantitative somatosensory testing of subjects with chronic post traumatic headache: implications on its medication. Eur J Pain 2010;14(9):924-31.

[9] Solomon S. Post traumatic headache: commentary: an overview. Headache 2009;49(7):1112-5.

[10] Bigal ME, Lipton RB. Concepts and mechanisms of migraine chronification. Headache 2008;48(1):7-15.

[11] McAllister TW, Saykin AJ, Flashman LA, et al. Brain activation during working memory 1 month after mild traumatic brain injury: a functional MRI study. Neurology 1999;53(6):1300-8.

[12] Mandel S. Minor head trauma may not be minor. Postgraduate Med J 1989;85:213-5.

[13] De Benedittis G, De Santis A. Chronic post-traumatic headache: clinical, psychopathologic features and outcome and determinants. J Neurosurg Sci 1983;27(3):177-86.

[14] Schwartz BS, Stewart WF, Simon D, et al. Epidemiology of tension-type headache. JAMA 1998;279(5):381-3.

[15] Haas DC. Classification of post traumatic headache. Cephalalgia 1995;15:162.

[16] Lew HL, Lin PH, Fuh JL, et al. Characteristics and treatment of headache after traumatic brain injury: a focussed review. Am J Phys Med Rehabil 2006;85(7):619-27.
[17] Natoli JL, Manack A, Dean B, et al. Global prevalence of chronic migraine: a systematic review. Cephalalgia 2010;30(5):599-609.

[18] Sjaastad O, Fredriksen TA, Sand T, et al. Unilaterality of headache in classic migraine. Cephalalgia 1989;9(1):71-7.

[19] Gfeller JD, Chibnall JT, Duckro PN. Post-concussion symptoms and cognitive functioning in posttraumatic headache patients. Headache 1994;34(9):503-7.

[20] Merskey H, Bogduk N. Classification of pain. Description of chronic pain syndromes and definition of pain terms. $2^{\text {nd }}$ edn. Seattle: International Association for the Study of Pain 1994:64-5.

[21] De Kruijk JR, Leffers P, Menheere PP, et al. Prediction of post traumatic complains after mild traumatic brain injury: early symptoms and biochemical markers. J Neurol Neurosurg Psychiatry 2002;73(6):727-32.

[22] Yilmaz T, Roks G, de Koning M, et al. Risk factors and outcomes associated with post traumatic headache after mild traumatic brain injury. Emerg Med J 2017;34(12):800-5.

[23] Census 2011. Chapter 6, State of literacy 2011: p. 14. Government of India.

[24] Report of the fifth annual employmentunemployment survey 2015-2016. Government of India: Ministry of Labour and Employment.

[25] Asian Development Bank, Retrieved 16 Aug 2017.

[26] Stovner LJ, Schrader H, Mickeviciene D, et al. Headache after concussion. Eur J neurol 2009;16(1):112-20.

[27] Russell MB, Ostegaard S, Bendtsen L, et al. Familial occurrence of chronic tension type headache. Cephalgia 1999;19(4):207-10.

[28] Bartleson JD, Cutrer FM. Migraine update. Diagnosis and treatment. Minn Med 2010;93(5):36-41. 